\title{
The Survival Of All Ceramic Crowns With A Zirconia Core (Lava ${ }^{\mathrm{TM}}$ )
}

Jiang Dai, Ling Xiao, Chen Dong, Fei Liu, Gang wei *

*Corresponding Author : Gang wei, Department of Pediatric Dentistry, china.

Received date: February 20,2019;Accepted date : March 10,2019; Published date: March 15,2019.

Citation: Gang wei, The Survival Of All Ceramic Crowns With A Zirconia Core (Lava ${ }^{\mathrm{TM}}$ ), J Dentistry and Oral Maxillofacial Surgery. I Doi: http://dx.doi.org/ 10.31579/Jdos.2019/011

Copyright : (C) 2019 Gang wei. This is an open-access article distributed under the terms of The Creative Commons Attribution License, which permits unrestricted use, distribution, and reproduction in any medium, provided the original author and source are credited.

\section{Abstract}

Background: Few studies have assessed the survival of restorations and particularly zirconia based crowns in the restoration of the severely worn dentition. This study aimed to determine survival and factors associated with failure in anterior teeth worn through to dentine restored with Lava ${ }^{\mathrm{TM}}$ crowns.

Methods: A convenience sample of 30 participants ( 27 male, 3 female) had 161 Lava $^{\mathrm{TM}}$ crowns placed by one specialist clinician in a hospital setting. Follow-up was over a median 72 month period.

Results: Of the 161 Lava $^{\text {TM }}$ crowns only 25 failed (15.5\%) in 15 individuals up to 84 months for the longest case. Major failures were total debond $(\mathrm{N}=7)$ or minor delamination chips within the veneer ceramic layer $(\mathrm{N}=18)$. The mean time to first failure was 25.23 months. The Kaplan Meier survival plot estimated the Mean Survival Time for the crowns to be 74 months $(95 \% \mathrm{Cl} 70.4,77.3)$. Failures by subject were not associated with an increase in Occlusal Vertical Dimension using a Dahl approach but were associated with an edge-to-edge incisal relation $(p<0.05)$, attrition $(p<0.05)$ and bruxism $(p<0.005)$.

Conclusion: The Kaplan Meier survival plot estimated the Mean Survival Time for the crowns to be 74 months. The high loads in bruxism result in increased risk of fracture or debond.

Clinical relevance: A protective bi-laminar splint is thus advisable. Nonetheless, application of zirconia based crowns in a difficult clinical situation such as bruxism can be a successful treatment modality.

Keywords: Tooth wear, bruxism, attrition, erosion, zirconia, survival

\section{Introduction}

Dental attrition is used to describe wear of tooth surfaces rubbing directly against each other secondary to parafunctional muscle activity also known as bruxism. The equivalent tribological term is two-body abrasion [1]. The term, tooth wear, includes acid erosion and abrasion as well as attrition. Mechanical rubbing action other than from teeth such as habits like chewing pencils or pipe smoking is potentially abrasive. The net result can manifest as worn teeth, which is an increasing dental problem. The recent UK Adult Dental Health Survey (ADHS) reported that $77 \%$ of examined adults had wear with $15 \%$ showing moderate wear and $2 \%$ having severe tooth wear [2]. The wide range of risk factors depend on the mode of wear but dietary acids and gastric acid are implicated in erosion and attrition is secondary to stress-related bruxism or clenching and grinding. Tooth wear is also age related such that more than $80 \%$ of the over 50 -year olds in the UK ADHS exhibited tooth wear [2]. It is, therefore, a common problem and many patients seek treatment primarily to improve appearance although sensitivity and functional problems may occur.

Worn short teeth present a unique restorative challenge. Insufficient clinical crown height for the retention of conventionally cemented crowns means alternative methods of restoration are required. Moreover, the loads imposed across teeth during para-function or bruxism are extremely high such that conventional feldspathic porcelain-fused to metal crowns are likely to fail [3]. Bonding to the remaining tooth structure to improve retention may provide a solution to this problem. The performance of all-ceramic crowns placed to restore worn teeth has hardly been investigated. A $6 \%$ failure rate was reported over four years in a small sample of 59 dentine bonded crowns placed in 16 patients [4]. The study period was recognised to be short and survival data was not stated [4]. A recent evidence based search to determine the best way to restore worn teeth resulted in no systematic reviews being found and concluded by stating that it is not known which restorative option was preferable in terms of longevity, preservation of vitality or minimisation of opposing tooth wear [5].
The main presenting complaint of patients with worn teeth is of poor aesthetics [6]. Restoration of the worn dentition, particularly from attrition when teeth are under extreme load during bruxism, is particularly challenging. All-ceramic crowns can maximise aesthetics and can be bonded to remaining tooth structure even if compromised. The two groups of all-ceramic crowns fall into the low strength, etchable glass based ceramics or high strength, non-etchable alumina or zirconia based ceramics. The former are traditional feldspathic ceramic or newer materials such as IPS Empress/emax (Ivoclar Vivadent, Liechtenstein), Finesse (Dentsply Ceramco, PA USA) and Authentic (Jensen CT, USA) and the latter include Procera (NobelBiocare, Sweden), Lava (3M Espe, MN, USA), In-Ceram (Vita, Germany) and Zircon (DCS, Switzerland).

The inherent strength of zirconia cores may be advantageous in the bruxist although the ceramming layer is the weak link. The application of high strength zirconia cored crowns for restoration of worn anterior teeth is lacking. This study aimed to determine survival of all ceramic crowns with a zirconia core (Lava, 3M Espe, St. Paul, MN) in a group of patients with tooth wear.

\section{Methods}

Tooth wear patients were referred to Liverpool University Dental Hospital for a specialist opinion. To be included in the study, participants were generally fit and healthy. The exception to this was a history of bulimia nervosa or gastric reflux as these were known risk factors associated with acid erosion and patients with these clinical conditions were included. Exclusion criteria were an inability to attend for annual follow-up and periodontal disease. This is therefore a convenience sample or case series not necessarily representative of all subjects with tooth wear. Furthermore, all cases were of an advanced nature defined as $>30 \%$ exposed dentine on any single surface [7]. All patients completed a thorough examination and were questioned about exposure to known risk factors. Dietary risk factors had to be brought under control prior to being enlisted into the study. A significant proportion of patients exhibited classic signs of bruxism such as mating wear facets in lateral excursions. 
The incisal relationships were recorded according to the British Standards classification [8] as either class I, II, III or edge-to-edge. The latter was seemingly a common presentation in males with severely worn incisors. The presence or absence of an inter-occlusal space between the anterior teeth in Inter-Cuspal Position (ICP) or Centric Occlusion (CO) was important to note as lack of space indicated the occurrence of dento-alveolar compensation and the need to increase the Occlusal Vertical Dimension using the Dahl concept $[9,10]$ but not using an appliance as originally described. The onestage Dahl procedure has been advocated and reviewed [11].

The number of teeth to be restored was determined by mutual agreement between the dentist and patient. The teeth had to be caries free and the peri-apical status was checked by radiographs. Preparation of the teeth involved standard procedures using high speed diamond burs and production of a narrow circumferential chamfer finish line at or slightly above the gingival margin. Axial reduction resulted in a taper of between $5-10^{\circ}$ but height reduction was not necessary given that the teeth were already short. All contact points were broken through. Depending on the severity of wear and which surfaces were involved, dentine was exposed on at least one surface, typically incisal and/or palatal aspects. All subsequent impressions were taken in low viscosity addition cured silicone (Provil ${ }^{\circledR}$, Dentsply) placed in a custom made tray. The impressions were sent to Techceram Ltd, (Shipley, BD17 7SW, UK) an accredited Lava ${ }^{\circledR}$ centre, for scanning of the dies and milling of the zirconia cores, which were thicker than usual at $1 \mathrm{~mm}$ thickness. The zirconia cores were returned to the hospital where the in-house trained laboratory ceramic technician completed the build-up and firing of the ceramming layers using the approved Lava ${ }^{\circledR}$ ceramic. An important design feature of the crowns was the need to minimise tensile or nonaxial forces and keep the crown under compression in the long axis during anterior guidance. This was done by provision of shallow guidance, thicker than normal incisal edges and shorter crowns than the originals, yet increasing crown height to improve aesthetics [12].

The fit surface of the crowns was roughened using CoJet Sand ${ }^{\circledR} 30 \mu$ powder at 2-3 bar (30-45psi) for 15 seconds as per manufacturer's instructions and all crowns were bonded with RelyX Unicem ${ }^{\circledR}(3 \mathrm{M}$ Espe, St.Paul, MN). Dental dam isolation was not used when bonding the crowns as adequate isolation was gained anteriorly with cotton wool rolls. Excess resin was removed with a bristle brush and an initial light cure was carried out at the margins of the crowns. Removal of excess resin and polishing was carried out using ultra fine diamond burs. Most patients had crowns placed on all four upper incisors and several had the upper canines included also. The anterior guidance was developed on a semi-adjustable articulator (Denar Mk II) to provide group function on protrusion with even contacts on all the crowns in order to distribute loads evenly, albeit on the anterior teeth only as the posterior teeth were taken out of contact in the Dahl approach. All participants were invited for annual follow-up and all the crowns were assessed according to modified USPHS criteria as previously described [4,13]. Variables were recorded on a database and statistical analysis performed using SPSS v19.

\section{Results}

A total of 30 participants with a mean age of 45 years (SD 13.0) had $161 \mathrm{Lava}^{\mathrm{TM}}$ crowns bonded onto worn anterior teeth (Table 1). The number of crowns placed according to incisal classification is shown in Table 2. Thirteen subjects were primarily diagnosed with erosion, 11 with attrition and 6 had multiple aetiology (Table 3). A total of 161 Lava crowns were bonded in the 30 subjects with a modal number of 6 restored teeth for each subject. This was generally the upper six anterior teeth. The mean length of time the crowns were in place was 66 months (sd 13months) with a range from 34 to 86 months. Failed crowns were those classified with complete debond, fracture or chip. Of the $161 \mathrm{Lava}^{\mathrm{TM}}$ crowns only 25 failed (15.5\%) in 15 subjects. The mean time to first failure was 25.3 months. None of the subjects developed caries under any of the crowns during the observation period.

\begin{tabular}{|c|c|c|c|}
\cline { 1 - 3 } Number of subjects & Mean age (years) & Std deviation & \multicolumn{1}{|}{} \\
\cline { 1 - 3 } Male & 27 & 46.15 & 13.00 \\
\hline Female & 3 & 34.33 & 8.62 \\
\hline Total & 30 & 44.97 & 13.03 \\
\hline
\end{tabular}

Table 1 : Mean age of subjects.

\begin{tabular}{|l|l|l|}
\hline Incisor relation & Number of patients & Number of Lava \\
\hline Class 1 & 13 & 72 \\
\hline Class 3 & 2 & 10 \\
\hline Edge-to-edge & 15 & 79 \\
\hline Total & 30 & 161 \\
\hline
\end{tabular}

Table 2: Number of $\mathrm{Lava}^{\mathrm{TM}}$ crowns placed in different incisal relations.

The Kaplan-Meier survival plot for all the 161 crowns is shown in Figure 1. The mean survival time was estimated to be 74 months $(95 \%$ CI 70.4 , 77.3).

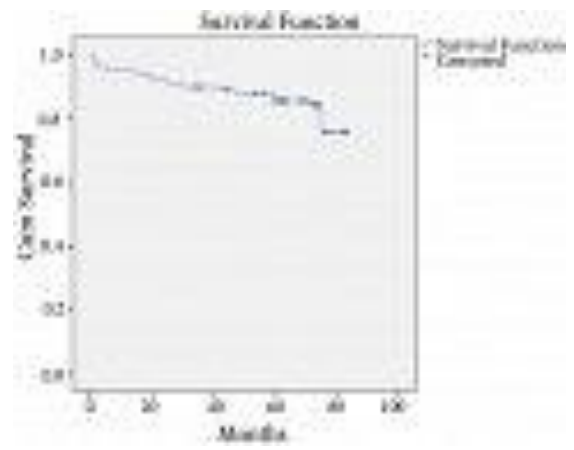

Figure 1 : Kaplan-Meier survival plot for all $161 \mathrm{Lava}^{\mathrm{TM}}$ crowns.

Figures $2 \mathrm{~A}$ and $2 \mathrm{~B}$ show a Lava ${ }^{\mathrm{TM}}$ crown case in a middle aged male with significant loss of coronal height and resultant poor aesthetics preoperatively. The image in Figure 2B shows the labial presentation with excellent aesthetics. Patients are informed of the planned alteration in OVD when applying the Dahl approach. Figures 3A and 3B show a lower attrition case managed with Lava ${ }^{\mathrm{TM}}$ crowns. Subtle characterisation of the ceramic allowed a hint of dentine to be introduced at the request of the patient as he did not wish to undergo a radical or noticeable change.

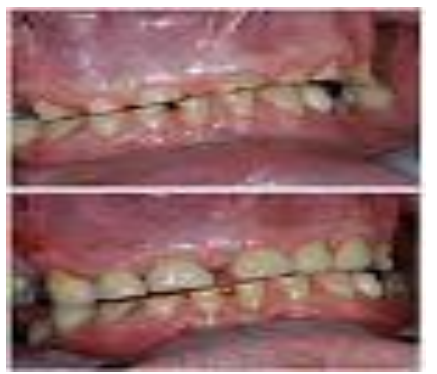

Figure 2 : Labial images of severe tooth wear, primarily attrition in a 49year-old male.

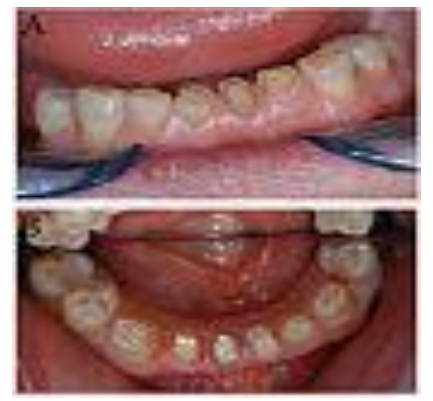

Figure 3: The lower anterior teeth exhibit flat, angulated wear facets indicative of attrition, secondary to bruxism. 


\section{Discussion}

This study aimed to estimate mean survival time and factors associated with failure, defined as debond and delamination of the ceramming layer. The preponderance of males in this study reflects the well documented male dominance in tooth wear. The reason for this is not known but probably is related to dietary factors such as a greater intake of acidic foods and drinks as well as greater functional (masticatory) forces and parafunctional (bruxist) loads. The females were seen at a significantly younger mean age (34 years) than the males (46 years), which reflects the general trend for hospital attendance plus the established common complaint of poor appearance in tooth wear patients being the driver to seek treatment [6]. The subjects in this study exhibited severe anterior tooth wear as all the teeth restored with Lava ${ }^{\mathrm{TM}}$ crowns had exposed dentine. Several had very little remaining tooth substance, having lost more than $2 / 3$ of the coronal tooth structure. Only one previous study reported on the performance of 48 standard feldspathic dentine crowns bonded in fewer than 16 tooth wear subjects over 4 years (59 were bonded in 16 patients but 48 crowns were followed up) [4]. The resultant $6 \%$ crown failure rate in a small sample size should be treated with caution and the risk factors for failure were not determined. The $15.5 \%$ failure rate of Lava ${ }^{\mathrm{TM}}$ crowns reported here is higher but the study is longer by 3 years and the cases probably more severe. Statistical analysis at the restoration level is difficult to interpret as it is not independent data since failures can be nested within subjects. Analysing data at the subject level is preferable but requires a larger cohort of subjects. Limitations of this study are the relatively small sample size and the generalisability to all patients with tooth wear although follow-up was longer than in previous studies.

Bonding zirconia to tooth substance is deemed not possible as the material is not etchable, although sand blasting may result in a rough/altered surface allowing some resin infiltration. Luting agents that employ 10-MDP (10-methacryl oxy decyl dihydrogen phosphate) as functional monomers have better results compared to Bis-GMA based agents [14]. That $84.5 \%$ of the Lava crowns functioned well over a 7-year period in such a challenging environment indicates that these crowns are feasible even with limited inherent resistance and retention form of the preparations. It is not surprising that failure was associated with bruxism. The maximum bite force in males is significantly greater than in females and higher posteriorly compared to anteriorly [15]. The load imposed across teeth in subjects with attrition was reported to be $911 \mathrm{~N}$ in the molar region and $569 \mathrm{~N}$ in the incisor region, which was significantly greater than normal [16]. To improve axial load distribution of the anterior teeth and reduce unfavourable shear forces, the incisal edges were designed to be thicker than normal so that the ceramic was under compression rather than tension [12]. This was particularly appropriate in half the subjects with an edge-to-edge incisal relationship. An increase in OVD by using the Dahl approach was needed in 23 out of the 30 subjects and this would only serve to increase the load on the Lava ${ }^{\mathrm{TM}}$ crowns and thus the likelihood of fracture. Why a higher number of failures occurred with an edge-to-edge incisal relationship and attrition/bruxism but not with an increase in OVD is not clear. Both the incisal relationship and bruxism were pre-existing conditions prior to the crowns being fitted whereas the OVD change was not. The assumption that an increase in OVD leads to deleterious or increased loads on those teeth may be erroneous. Although a significant proportion of failed crowns occurred in the bruxists, it would appear that in some subjects Lava crowns are viable. It should be noted that in the $50 \%$ of subjects who had a failure, many had multiple crowns which were successful. Of the 7 individuals with a failed crown, only one was female although 3 females were classed as bruxists. Within the 25 failed crowns, 7 suffered major failure or total debond (USPHS code 3) and 18 exhibited minor chips or delamination within the overlying ceramic (USPHS code 2). The subjects in this latter group accepted the chipped crowns and they were not replaced. Ceramic fractures in conventional porcelain fused-to-metal crowns would be less acceptable as metal is often visible.
Furthermore, in a wet and acidic environment, bond strength may deteriorate. This is especially relevant in this study as 13 subjects were primarily diagnosed with acid erosion. Despite appropriate advice to cut out or reduce acid intake, it would be important to know that the bond between zirconia and tooth does not deteriorate in acidic milieu as not all patients comply with advice. After thermocycling and storage in saliva at pH3.5, of 3 resins (Calibra, Panavia F2 and Unicem) bonded to zirconia (Cercon, Dentsply) the shear bonds of the latter two proved durable and performance was satisfactory [17].

The preparation of the teeth was fairly minimal and margins were supragingival. This optimised plaque control and gingival inflammation was not a feature in the majority of patients. The marginal fit of zirconia based cores was reported to be below $60 \mu$ for either in-house laboratory manufacture or centralised milling [18]. The milling and firing of the zirconia cores was carried out at an approved milling centre in order to maximise quality control during manufacture. The USPHS criteria are well known and include ratings for marginal adaptation, discolouration and colour match. All these were scored 0 or 1 . None of the participants had secondary caries during the period of follow-up. Occlusal planning in all cases was carried out on a programmed semi-adjustable articulator (Denar Mark II). Whenever possible anterior group function was incorporated in the occlusal scheme but it should be recognised that imbrications and the unevenness found in the worn dentition prevented even contacts being developed in some cases. Of the 30 subjects, 23 required a Dahl approach whereby the crowns were placed at an increased OVD, taking the posterior teeth out of contact. Posterior contacts were reestablished within a year in all subjects as checked by shimstock foil between opposing posterior teeth bilaterally. Given that bruxism was associated with crown failure, reduction of the excessive loads on newly restored teeth would be advisable. The author recommends the bi-laminar or dual laminate splint, which has a hard outer shell bonded to a soft vinyl inner fitting surface. This is more durable than a soft splint and is generally best located on the opposite arch to the crowned arch. The $3 \mathrm{~mm}$ blank is formed on a cast in the usual manner by way of vacuum and heat. The resultant splint should be a minimum of $2 \mathrm{~mm}$ thickness. Wear of the opposing natural dentition was not assessed although anecdotally this did not appear to be an issue during the period of the study. An edge-to-edge incisal relationship occurred in $15(50 \%)$ of subjects. It is interesting to speculate that was not the original incisal relationship but resulted when bruxists with a class I incisal relation wore the anterior teeth, losing the original overbite in the attrition process and thus becoming edge-to-edge. Certainly this type of incisal relation appears to be common in tooth wear cases.

The management of tooth wear can be difficult. The treatment strategy to adopt in a primarily publicly funded service should be cost effective, low maintenance and reasonably durable. The UK has a publicly funded National Health Service and most dentistry is provided by general dental practitioners working on behalf of the state with a subsidised patient charge based on a three band fee scale in England and Wales. Scotland and Northern Ireland use a fee-per-item of treatment. Zirconia crowns are not available on the current NHS dental list of materials for crowns. Many UK dentists therefore refer patients to local dental hospitals, where care is provided without charge to the patient as hospital funding is from a separate, central budget. The introduction of zirconia into mainstream dental provision is an aspiration and this study has shown that it has a place in the management of severe tooth wear.

\section{Conclusion}

This study aimed to determine survival of all ceramic crowns with a zirconia core (Lava, 3M Espe, St. Paul, MN) and factors related to failure in a group of patients with tooth wear. Only 25 crowns failed $(15.5 \%)$ in 15 individuals up to 84 months for the longest case. The Kaplan Meier survival plot estimated the Mean Survival Time for 161 zirconia based crowns placed in 30 individuals to be 74 months (95\% CI 70.4, 77.3). Failures such as debond or delamination of the veneering ceramic were not associated with an increase in Occlusal Vertical Dimension using a Dahl approach, but were associated with an edge-to-edge incisal relation, attrition and bruxism. 


\section{References}

1. Mair LH. Wear in dentistry--current terminology. J Dent. 1992; 20:140-4

2. The Health and Social Care Information Centre. Adult dental health survey 2009. London: Department of Health. 2010.

3. Kelleher MG, Bomfim DI and Austin RS. Biologically based restorative management of tooth wear. Int J Dent. 2012; 2012:742509

4. Burke FJ. Four year performance of dentine-bonded allceramic crowns. Br Dent J. 2007; 202:269-73.

5. Hurst $D$. What is the best way to restore the worn dentition? Evid Based Dent. 2011; 12:55-6

6. Wazani BE, Dodd $\mathrm{MN}$ and Milosevic A. The signs and symptoms of tooth wear in a referred group of patients. $\mathrm{Br}$ Dent J. 2012; 213:E10.

7. Smith BG and Knight JK. A comparison of patterns of tooth wear with aetiological factors. Br Dent J. 1984; 157:16-9.

8. Mitchell L. The aetiology and classification of malocclusion 3rd ed. In Mitchell L (Ed)., An introduction to orthodontics. 2007; 8-13.

9. Dahl BL, Krogstad O and Karlsen K. An alternative treatment in cases with advanced localized attrition. J Oral Rehabil. 1975; 2:209-14.

10. Dahl BL and Krogstad O. The effect of a partial bite raising splint on the occlusal face height. An x-ray cephalometric study in human adults. Acta Odontol Scand. 1982; 40:17-24.
11. Poyser NJ, Porter RW, Briggs PF, Chana HS and Kelleher MG. The Dahl Concept: past, present and future. Br Dent J. 2005; 198:669-76.

12. Mizrahi B. The Dahl principle: creating space and improving the biomechanical prognosis of anterior crowns. Quintessence Int. 2006; 37:245-51.

13. Ryge G. Clinical criteria. Int Dent J. 1980; 30:347-58.

14. Wolfart M, Lehmann F, Wolfart S and Kern M. Durability of the resin bond strength to zirconia ceramic after using different surface conditioning methods. Dent Mater. 2007; 23:45-50.

15. Helkimo E, Carlsson GE and Helkimo M. Bite force and state of dentition. Acta Odontol Scand. 1977; 35:297-303.

16. Waltimo A, Nystrom $M$ and Kononen $M$. Bite force and dentofacial morphology in men with severe dental attrition. Scand J Dent Res. 1994; 102:92-6.

17. Geramipanah F, Majidpour M, Sadighpour L and Fard MJ. Effect of artificial saliva and $\mathrm{pH}$ on shear bond strength of resin cements to zirconia-based ceramic. Eur J Prosthodont Restor Dent. 2013; 21:5-8.

18. Beuer F, Korczynski N, Rezac A, Naumann M, Gernet W and Sorensen JA. Marginal and internal fit of zirconia based fixed dental prostheses fabricated with different concepts. Clin Cosmet Investig Dent. 2010; 2:5-11. 